\title{
Atropine Penalization Versus Occlusion Therapies for Unilateral Amblyopia after the Critical Period of Visual Development: A Systematic Review
}

\author{
Daniel C. Osborne (D) - Kathryn M. Greenhalgh • Megan J. E. Evans • \\ Jay E. Self
}

Received: September 12, 2018 / Published online: October 16, 2018

(C) The Author(s) 2018

\section{ABSTRACT}

Introduction: Amblyopia therapy appears to be most effective in children under the age of 7 years, but results from randomized control trials (RCTs) have shown that occlusion therapy and/or atropine penalization therapy may improve visual acuity in an older age group. Which of these two therapies is the most effective with fewer adverse effects in an older age group has not yet been agreed upon.

Methods: We systematically searched the literature for RCTs that compared atropine penalization therapy and occlusion therapy in terms

Enhanced Digital Features To view enhanced digital features for this article go to https://doi.org/10.6084/ m9.figshare.7172411.

Electronic supplementary material The online version of this article (https://doi.org/10.1007/s40123018-0151-9) contains supplementary material, which is available to authorized users.

D. C. Osborne $(\bowtie) \cdot$ M. J. E. Evans

Orthoptic Department, University Hospital

Southampton NHS Foundation Trust,

Southampton, UK

e-mail: daniel.osborne@uhs.nhs.uk

K. M. Greenhalgh

Orthoptic Department, Hampshire Hospitals NHS

Foundation Trust, Winchester, UK

\section{J. E. Self}

Clinical and Experimental Sciences, Faculty of

Medicine, University of Southampton,

Southampton, UK of their visual acuity outcomes and adverse events and performed a meta-analysis on the visual acuity data obtained. The adverse effects reported and their implications for clinical practice are discussed.

Results: Two RCTs were identified, with the authors of both concluding that there was no detectable difference between the two therapies for the age groups they studied. The mean difference between atropine penalization and occlusion therapies was calculated to be -0.01 logMAR (95\% confidence interval -0.07 to 0.03 logMAR) in favor of occlusion therapy, and no statistical difference between the two groups was detected $(P=0.45)$. Neither study detected a marked difference in terms of reported adverse effects from the two interventions.

Conclusion: Based on the results of our metaanalysis we conclude that there is no difference in visual acuity outcomes between atropine penalization therapy and occlusion therapy after 17 to 24 weeks of treatment in children aged 7-12 years. Further evidence to determine the efficacy of amblyopia therapy for an older patient population is required before studies comparing atropine penalization and occlusion therapy in patients older than 12 years can be performed. Atropine penalization therapy may cause more frequent minor adverse effects, such as light sensitivity, but in the clinical setting this needs to be balanced with the potential practical benefits of twice-weekly eye drops versus daily occlusion. 
Funding: The funding for this study was provided by the National Institute for Health Research (NIHR) and Health Education England (HEE).

Plain Language Summary: A plain language summary is available for this article.

Keywords: Amblyopia; Atropine penalization therapy; Occlusion therapy; Pediatric ophthalmology; Visual development

\section{PLAIN LANGUAGE SUMMARY}

If sight is impaired in one eye during early life a patient may unconsciously and involuntarily choose to use the unimpaired eye. The lack of use of one eye results in that eye not learning to see properly. This condition is commonly referred to as "lazy eye"; the medical term for it is amblyopia. Amblyopia is treated by forcing the patient to use their lazy eye through "penalizing" the good eye by either covering it up for a prescribed number of hours per day (occlusion therapy) or by blurring it with eye drops (atropine penalization therapy).

The efficacy of the chosen treatment is assessed at various time points by asking patients just how small an object they can see with the lazy eye; if the treatment is working, the patient will slowly begin to see smaller and smaller objects with the lazy eye. The standard test used to grade how small an object an eye can see is called the visual acuity test. Children aged $<8$ years are normally given either occlusion therapy or atropine penalization therapy, and previous research has shown that, in terms of visual acuity outcome, these two treatments work equally well in children in this age group. It is also known that these treatments can work in children up to 12 years of age and that they may also work for children aged between 13 and 17 years of age. However, whether the clinical outcome of occlusion therapy is equal to or better or worse than that of atropine penalization therapy in children aged $\geq 8$ years is as yet unknown.

We therefore performed a systematic search of the current literature to identify studies that had been conducted with the specific aim of determining whether atropine penalization therapy or occlusion therapy is the better therapy for children older than 7 years. We identified two studies in which these two therapies had been compared. The authors of both studies concluded that based on their respective results there was no measurable difference between the atropine penalization and occlusion therapies for amblyopia in children aged $>7$ years. The two studies were graded as being of high and moderate quality, respectively, and we performed further analysis on the combined results of these two studies.

Our analysis found no difference between the atropine penalization and occlusion therapies for the age group the studies reported on (age 7-20 years). Patients receiving atropine penalization therapy may more frequently report minor eye complications, such as light sensitivity, compared to those having occlusion therapy. However, these minor reactions must be balanced with the benefits atropine penalization therapy has over occlusion therapy, such as increased patient compliance with the therapy.

\section{INTRODUCTION}

Amblyopia refers to the maldevelopment of visual function of one or both eyes in the critical period of visual development, which continues from birth through the first 7-8 years of life $[1,2]$. It arises secondary to any ocular pathology that disrupts the function of one or both eyes during this critical period and is characterized by an inability to resolve small letters, i.e., a reduced visual acuity.

In the case of symmetrical bilateral amblyopia, effective management of the primary ocular pathology within the critical period time frame results in the improvement of visual acuity [3]. Conversely, in the case of unilateral amblyopia, visual acuity may not improve once the primary ocular pathology is managed [4]. The most common primary causes of unilateral amblyopia include anisometropia, strabismus, or a combination of both. Unilateral amblyopia is treated in one of two ways in the UK [5]: 
1. Occlusion therapy, which consists in patching the fellow "sound" eye for a prescribed period of time per day, typically, between 2 and $6 \mathrm{~h}$ depending on the visual acuity at diagnosis [6].

2. Atropine penalization, which consists in the instillation of $1 \%$ atropine eye drops twice weekly into the sound eye, thereby blurring its visual acuity.

If a patient has a moderate reduction in baseline visual acuity and treatment is started before age 7 years there is no difference between option 1 and option 2 in terms of visual acuity outcome and rate of complications [7]. However, for a patient with poor baseline visual acuity, i.e., severe amblyopia, evidence comparing atropine penalization therapy and occlusion therapy is less robust [8]. The most significant factor in determining visual acuity outcome is adherence to the prescribed occlusion therapy protocol [9]. It has often been found that children in a clinical setting reject occlusion therapy and as a result remain under review in the orthoptic department for many years before being discharged with unfavorable visual acuity outcomes [10].

Orthoptists and pediatric ophthalmologists are taught that unilateral amblyopia must be managed before the age of 7 years. The efficacy of occlusion therapy and atropine penalization therapy in the over 7-year age group is not understood [11, 12]. Randomized control trials (RCTs) have explored treating unilateral amblyopia in patients older than 7 years by comparing no treatment versus therapy [13] and found that therapy for amblyopia in a postcritical period population is likely to improve visual acuity if atropine penalization or occlusion therapies have not been tried previously [14].

There are significant differences in the way amblyopia is managed in the UK versus German Speaking Countries (GSC), but a comparative study of European countries by Tan et al. found that visual acuity outcomes are comparable [5]. These authors reported that amblyopia is more aggressively managed in GSC compared to the UK in terms of continuing therapy into the over 7-year age group and the number of hours of occlusion therapy prescribed. National guidelines for the UK and Ireland published by the British and Irish Orthoptic Society [15] recommend the treatment of amblyopia with either occlusion or atropine penalization but do not reference age as a factor to be considered in the management of this condition. As such, this guideline reflects the weak evidence for continuing amblyopia therapy into adulthood and is supported by the literature narratively discussed above and explored in a Cochrane systematic review [11].

Large, multicenter RCTs have shown that atropine penalization therapy of the sound eye using $1 \%$ atropine eye drops twice weekly achieves visual acuity outcomes that are comparable to those achieved with part-time total occlusion therapy in patients aged 7 years and younger with moderate amblyopia [7, 16]. Studies by the same group have shown that visual acuity in an over 7-year age group diagnosed with isolated unilateral strabismic and/or anisometropic amblyopia can be improved with these therapies when compared to no treatment [13]. The same research group has also published some of its work comparing part-time total occlusion therapy to twice-weekly atropine penalization therapy in patients aged 7-12 years [14], in which they found no difference between atropine penalization using atropine $1 \%$ eye drops twice weekly and occlusion therapy in this age group.

Visual development is thought to end at approximately age 7 years [17], after which amblyopia therapy is likely to be less effective. This theory is based on extrapolations from studies on the macaque monkey $[1,2]$. If the theory is correct, isolated anisometropic and or strabismic amblyopia patients fall into one of two categories: (1) those aged $\leq 7$ years who are likely to respond to treatment and (2) those aged $>7$ years who are unlikely to respond to treatment. In the systematic review reported here, we were interested in the second group of patients, i.e., those aged $>7$ years diagnosed with isolated, unilateral strabismic and/or anisometropic amblyopia. The aim of our review 
was to compare atropine penalization and occlusion therapies for this group of patients.

\section{METHODS}

We designed and completed a systematic review of the literature using the following methodology in concordance with the PRISMA (Preferred Reporting Items for Systematic Reviews and Meta-Analyses) statement [18]. This review is registered with the International Prospective Register of Systematic Reviews (PROSPERO) (registration no. CRD42018089324). It is based on previously published studies and does not contain any studies with human participants performed by any of the authors.

Inclusion Criteria To be included in this review, studies had to meet the criteria for participants, intervention, comparator, outcome measure, and design set out in Table 1.

Search Strategy We performed a search of the MEDLINE, EMBASE, and CENTRAL databases using the keywords summarized below and a the search strategy to identify randomized control trials proposed by Glanville et al. [19]. The Boolean search strategy is available in the Electronic Supplementary Material table. Our search was limited to publications available in the English language which were published before 12 April 2018. Keywords: amblyopia, refractive error, anisometropia, strabismus, atropine penalisation, patching.

Study Screening All studies identified in our literature search were initially screened by title. If no reason was found to exclude the study, the reviewer read the abstract; if again no reason was found to exclude the study, the full text was obtained for full text screening. Following the full text screening, the reviewer decided whether to include or exclude the study from this systematic review. This screening process was carried out by two reviewers independently (DO and $\mathrm{KG}$ ); in cases of disagreements between these two reviewers, resolution was achieved with discussion and input from a third reviewer, ME.

Critical Appraisal All included studies were critically appraised by the lead author (DO). The Critical Appraisal Skills Programme (CASP) toolkit [20] was used to rate the quality of evidence for each included study as "low", "moderate", or "high."

Data Extraction and Statistical Analysis The visual acuity outcomes were narratively discussed and presented in the Results section. Where appropriate, we used a forest plot to determine the overall effect from the included studies. For the purposes of our statistical analysis, visual acuity measures were converted into logMAR units. The reported mean improvement in visual acuity from baseline, the standard deviation (SD) of improvement from baseline, and sample sizes were used to calculate a 95\% confidence interval (CI) for each study. We combined the results from both studies to calculate a total mean difference and 95\% CI between occlusion therapy and atropine penalization therapy using Review Manager 5 (Cochrane, London, UK). The secondary outcome of this review, adverse reactions and adverse events, are discussed.

\section{RESULTS}

Our search strategy identified 1043 publications, of which 229 were duplicates. Of the remaining 814 publications, 789 were excluded based on the primary title and abstract screening; a further 23

Table 1 Summary of criteria for studies to be included in the systematic review

\begin{tabular}{ll}
\hline Factor & Criteria \\
\hline Participants & Children with a diagnosis of anisometropic, strabismic, or mixed anisometropic/strabismic amblyopia \\
Intervention & Atropine penalization therapy \\
Comparator & Part-time total occlusion therapy \\
Outcome measure & Best corrected visual acuity after a period of atropine or occlusion therapy \\
Design & Randomized control trial \\
\hline
\end{tabular}




\title{
PRISMA 2009 Flow Diagram
}

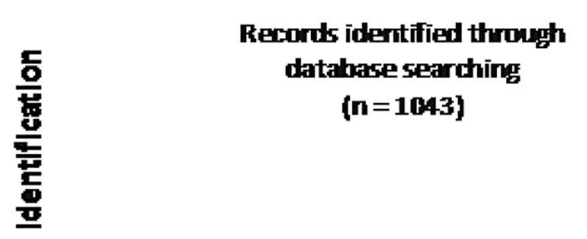
Additional reconds identified through other sources $(n=\mathbf{b})$

Reconks after dhplicates removed

( $\mathbf{n}=\mathbf{8 1 4}$ )

본
홍
훈

Records sireened

(n=814)

Full-text articles assersed

for elighility

(n=25)
Reconds exchuded

(n=789)

吾

\author{
Full-text articles exchuded, \\ with reasons \\ $(\mathbf{n}=\mathbf{2 3})$
}

$$
\begin{aligned}
& \text { Studies ircluded in } \\
& \text { qualitative syrthesis } \\
& \text { (n=2) }
\end{aligned}
$$

폴

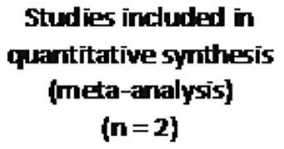

Fig. 1 PRISMA (Preferred Reporting Items for Systematic Reviews and Meta-Analyses) flowchart

were excluded after the full text eligibility screening. Ultimately, there were only two studies which met our criteria for critical appraisal and qualitative and quantitative data synthesis. A summary of the screening and eligibility process is summarized in the PRISMA flowchart [18] shown in Fig. 1, and a summary of the included studies is outlined in Table 2.

\section{Included Studies and Critical Appraisal}

Both studies included in the systematic review used a robust computer randomization process to randomly allocate participants to either an atropine penalization therapy group or an occlusion therapy group. For the Pediatric Eye Disease Investigator Group (PEDIG) [14] study, visual acuity assessors were blinded to the result of the randomization, but due to the nature of the intervention it was not possible to blind the participants; the masked assessors remained masked for $97 \%$ of the appointments. Neither the examiners nor the participants were blinded to the treatment protocol in the Menon et al. [21] study. In both studies a number of participants withdraw from the study prior to completion of the full follow-up and primary outcome data collection visit; the attrition rate 


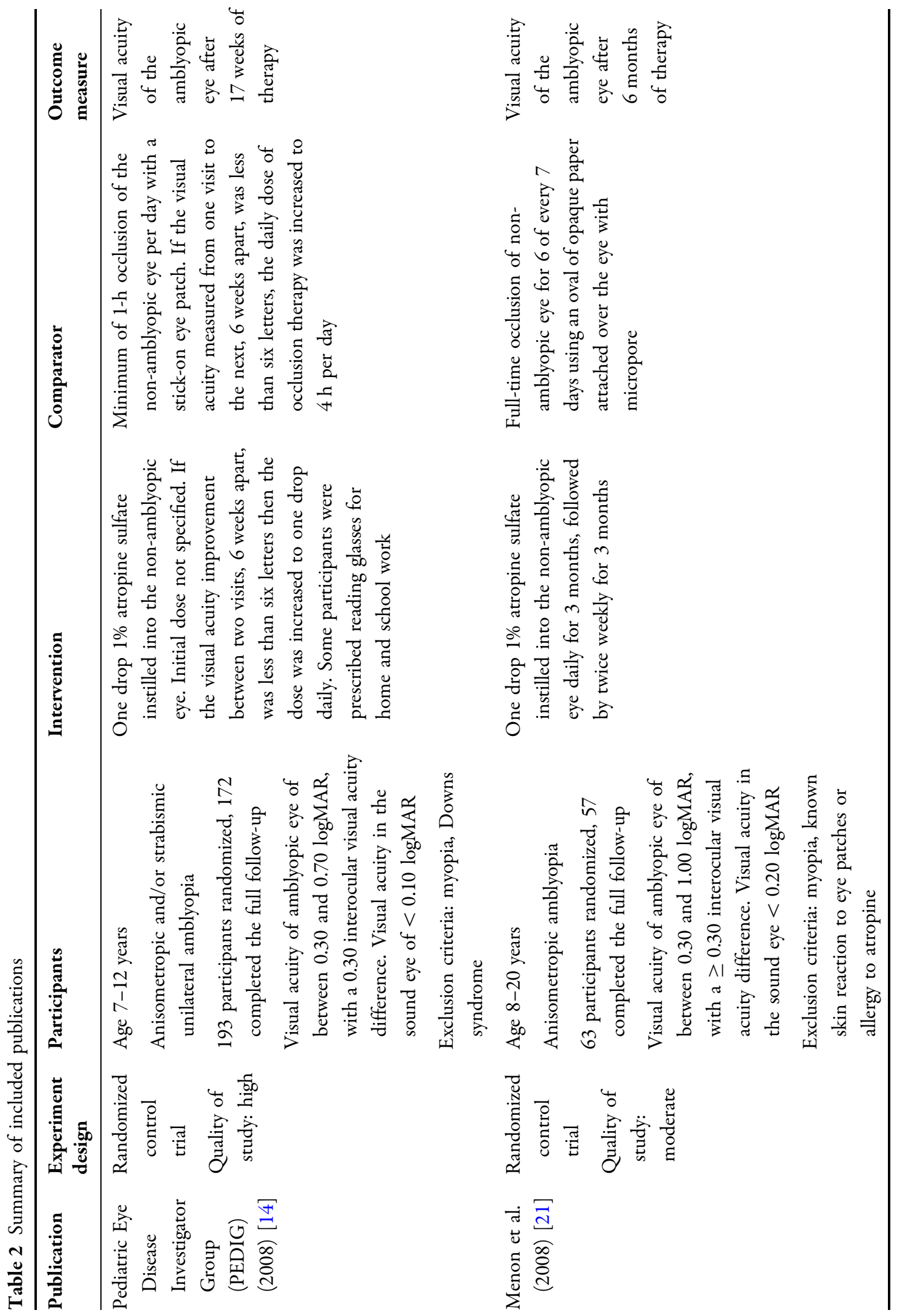


was the same for both the atropine penalization therapy and occlusion therapy groups. The quality of evidence in the PEDIG [14] study was graded as high, and that in the Menon et al. [21] study graded as moderate.

Both studies reported the baseline characteristics of the patients included in the respective study in tables. There are small differences of the characteristics of the participants between the intervention and comparator groups in both studies:

- PEDIG [14] had more participants with anisometropic amblyopia in the patched group (46/98 in occlusion therapy group versus 31/95 in the atropine penalization therapy group). However, anisometropic and strabismic amblyopia responded similarly to treatment.

- Menon et al. [21] reported a difference in the spherical equivalent refractive errors between the two groups, with the patched group being more hyperopic than the atropine group $(+4.24$ [SD 1.66] vs. +3.29 [SD 1.47], respectively). This difference is statistically significant $(P=0.027)$.

Visual acuity is measured using a variety of visual acuity testing protocols. Of these, the early treatment of diabetic retinopathy vision test (ETDRS) is considered to be the gold standard of visual acuity assessment [22], and the ETDRS values were used in this review.

Menon et al. [21] excluded all participants that had had more than 2 months of amblyopia treatment within the last 2 years. In contrast, PEDIG [14] included participants regardless of whether or not they had had previous therapy, but they ensured that the visual acuity of each patient with previous therapy was stable without atropine penalization or occlusion therapies for at least 4 weeks prior to the start of the study. In the PEDIG [14] study, 27 of the 95 patients receiving atropine penalization therapy and 27 of the 98 patients receiving occlusion therapy had had previous atropine and/or occlusion therapy for amblyopia in the past.

\section{Meta-Analysis}

We performed a statistical analysis of the primary visual acuity outcome data of the two studies [14, 21]. Visual acuity in both studies was measured using the ETDRS or electronicETDRS test charts. PEDIG [14] reported a mean improvement in visual acuity from baseline of 7.6 letters (0.152 logMAR) and 8.6 letters $(0.172$ $\log$ MAR) for atropine therapy and occlusion therapy, respectively. Menon et al. [21] reported a mean improvement in visual acuity from baseline of 2.34 lines (0.234 $\log$ MAR) and 2.38 lines (0.238 logMAR) for atropine and patching, respectively.

In our analysis a negative number is in favor of occlusion therapy and a value of 0 represents no difference between the two intervention groups. The total mean difference between the two interventions from the two studies was calculated to be $-0.01 \log$ MAR $(95 \% \mathrm{CI}-0.05$ to 0.02 ), indicating that there was no statistical difference between the two interventions $(P=0.45)$. We used a Chi-squared test for heterogeneity, which showed no statistically significant heterogeneity between the two studies $(P=0.68)$. These results are shown in Fig. 2.

\section{Adverse Events and Reactions}

Adverse events from both interventions were discussed in the text of both studies, and data were presented in tables. Menon et al. [21] asked participants about eye redness and itchiness, and participants in both groups reported itchiness; however, more participants in the atropine group reported red eye [8/28 (28.6\%) vs. $2 / 29(7.9 \%)$ for atropine and occlusion groups, respectively]. PEDIG [14] reported that no participants were diagnosed with reverse amblyopia during the course of the study; however, visual acuity of the sound eye worsened by 0.3 letters (0.006 logMAR) and 1.5 letters (0.03 $\log$ MAR) in the atropine and occlusion groups, respectively (mean difference between groups adjusted for baseline was 1.3 letters; 95\% CI 0.4-2.2 letters). In the PEDIG [14] study, patients in the atropine group reported ocular and systemic adverse events as follows: ocular adverse events (14/88 [15.9\%]), with the most common complaint being light sensitivity; tachycardia (1/88 [1.1\%]); dry mouth (1/88 


\begin{tabular}{|c|c|c|c|c|c|}
\hline Study or Subgroup & $\begin{array}{c}\text { Mean Difference } \\
\text { IV, Fixed, 95\% Cl [logMAR] }\end{array}$ & \multicolumn{4}{|c|}{$\begin{array}{c}\text { Mean Difference } \\
\text { IV, Fixed, 95\% CI [logMAR] }\end{array}$} \\
\hline Menon et al., 2008 & $-0.00[-0.06,0.06]$ & & & & \\
\hline Scheiman et al., 2008 & $-0.02[-0.07,0.03]$ & & & & \\
\hline Total $(95 \% \mathrm{Cl})$ & $-0.01[-0.05,0.02]$ & & & & \\
\hline \multicolumn{2}{|c|}{$\begin{array}{l}\text { Heterogeneity: } \mathrm{Chi}^{2}=0.17, \mathrm{df}=1(\mathrm{P}=0.68) ; \mathrm{I}^{2}=0 \% \\
\text { Test for overall effect: } Z=0.76(P=0.45)\end{array}$} & $\vdash_{-0.1}$ & $\begin{array}{l}-0.05 \\
\text { Favors patching }\end{array}$ & Favors atropin & 0.1 \\
\hline
\end{tabular}

Fig. 2 Forest plot showing no significant difference between occlusion and atropine penalization therapies. CI Confidence interval, $I V$ inverse variance

[1.1\%]); irritability and headache (1/88 [1.1\%]). The authors of this study also analyzed the change in angle of strabismus throughout the course of the study and reported "no differences between treatment groups in the number of participants who developed new-onset strabismus or had an increase or decrease in a preexisting strabismus." PEDIG [14] reported that no participants in either group developed any persistent, constant diplopia; Menon et al. [21] did not report any cases of diplopia through the course of their study.

\section{DISCUSSION}

Theories derived from invasive studies on macaque monkeys $[1,2]$ suggest that the critical period of visual development ends at approximately age 7 years, after which amblyopia therapies are unlikely to improve visual acuity in the amblyopic eye of human subjects. Scheiman et al. [13] robustly showed amblyopia can be treated in the 7- to 12-year age group compared to a control group and also showed that treatment of an older population aged 13-17 years may be possible with current occlusion and atropine penalization therapies. Innovative studies have also shown that if, in adulthood, a unilateral amblyopia patient suffers insult to vision in their sound eye their amblyopic eye may see improvement in visual acuity, suggesting amblyopia therapies may be effective in an adult age group [23].

The respective authors of the two trials identified in this review $[14,21]$ both concluded that there was no statistical difference between atropine penalization therapy and occlusion therapy in the samples they studied. We used a meta-analysis statistical technique to combine the results from the two studies to form a larger sample size with greater statistical power and found that the combined results do not allow us to reject the null hypothesis. The aim of our systematic review was to compare the visual acuity outcomes of atropine penalization and occlusion therapies in a post-critical period of visual development. Patients included in the two studies in our review ranged in age from 7 to 20 years; consequently, patients aged $>20$ years are not represented in our analysis; in fact we did not identify any studies for such patients aged $>20$ years. It should also be noted that the distribution of age in the 229 participants that completed follow-up in our review are not evenly spread between the two studies. The largest study in terms of sample size in this review was that of PEDIG [14], which did not include participants older than 13 years of age, resulting in a large number of 7- to 13-year-olds included in our review, but relatively few 14- to 20 -year-olds. This represents an evidence gap for amblyopia patients aged $>13$ years of age.

The secondary outcome of this review was adverse effects and events reported by the two studies. Amblyopia therapies are thought to carry a risk of causing intractable, constant, binocular diplopia when used for an older population. In total, 229 participants completed the full follow-up protocol for the respective studies with zero reports of any participant acquiring binocular diplopia throughout the course of the study. Atropine penalization therapy appears to be associated with more frequent minor ocular adverse events than occlusion therapy, with light sensitivity and periocular itchiness being the most common complaints reported by those receiving atropine 
penalization therapy and occlusion therapy, respectively.

\section{CONCLUSION}

Our systematic literature search identified two studies [14, 21] that compared visual acuity outcomes from atropine penalization and occlusion therapies for unilateral amblyopia in patients at a post-critical period of visual development. Both studies reported no detectable difference in terms of visual acuity outcomes after 17 weeks [14] and 6 months [21] of treatment. Our meta-analysis of the combined results from both studies found no difference between treatments in terms of visual acuity outcomes. However, patients with unilateral anisometropic and/or strabismic aged 14-20 years were not well represented in this review, and we were unable to identify any no data for the over 20-year-old patients. As such, there is a gap in the evidence base for atropine penalization therapy versus occlusion therapy for unilateral amblyopia patients aged $>$ 12 years. Atropine penalization therapy may result in more frequent minor adverse events, such as red eye and light sensitivity; however, due to the infrequency of these events, a larger sample size may be required to determine the likelihood of these complications.

\section{ACKNOWLEDGEMENTS}

Funding. This review was funded by the National Institute for Health Research (NIHR) and Health Education England (HEE). No funding was received for this study or publication of this article.

Authorship. All named authors meet the International Committee of Medical Journal Editors (ICMJE) criteria for authorship for this article, take responsibility for the integrity of the work as a whole, and have given their approval for this version to be published.
Disclosures. Daniel C. Osborne, Kathryn M. Greenhalgh, Megan J. E. Evans and Jay E. Self declare no personal or financial conflicts of interest.

Compliance with Ethics Guidelines. This review is based on previously published studies and does not contain any studies with human participants performed by any of the authors.

Open Access. This article is distributed under the terms of the Creative Commons Attribution-NonCommercial 4.0 International License (http://creativecommons.org/licenses/ by-nc/4.0/), which permits any noncommercial use, distribution, and reproduction in any medium, provided you give appropriate credit to the original author(s) and the source, provide a link to the Creative Commons license, and indicate if changes were made.

\section{REFERENCES}

1. Hubel DH. Laminar and columnar distribution of geniculo-cortical fibers in macaque monkey. J Comp Neurol. 1972;146(4):421-50.

2. Horton JC, Hocking DR. Timing of the critical period for plasticity of ocular dominance columns in macaque striate cortex. J Neurosci. 1997;17(10):3684-709.

3. Stewart CE, Moseley MJ, Fielder AR, Stephen DA. Refractive adaptation in amblyopia: quantification of effect and implications for practice. Br J Ophthalmol. 2004;88(12):1552-6.

4. Clarke MP, Wright CM, Hrisos S, Anderson JD, Henderson J, Richardson SR. Randomised controlled trial of treatment of unilateral visual impairment detected at preschool vision screening. Br Med J. 2003;327(7426):1251-4.

5. Tan JHY, Thompson JR, Gottlob I. Differences in the management of amblyopia between European countries. Br J Ophthalmol. 2003;87(3):291-6.

6. Wallace DK, Pediatric Eye Disease Investigator Group. A randomized trial to evaluate 2 hours of daily patching for strabismic and anisometropic amblyopia in children. Ophthalmology. 2006;113(6):904-12. 
7. Glaser SR, Matazinski AM, Sclar DM, Sala NA, Vroman CM, Tanner CE, et al. A randomized trial of atropine vs patching for treatment of moderate amblyopia in children. Arch Ophthalmol. 2002;120(3):268-78.

8. Repka MX, Kraker RT, Beck RW, Birch E, Cotter SA, Holmes JM, et al. Treatment of severe amblyopia with weekend atropine: results from 2 randomized clinical trials. J AAPOS. 2009;13(3):258-63.

9. Dean SE, Povey RC, Reeves J. Assessing interventions to increase compliance to patching treatment in children with amblyopia: a systematic review and meta-analysis. $\mathrm{Br} \mathrm{J}$ Ophthalmol. 2016;100(2):159-65.

10. Awan M, Proudlock FA, Grosvenor D, Choudhuri I, Sarvanananthan N, Gottlob I. An audit of the outcome of amblyopia treatment: a retrospective analysis of 322 children. $\mathrm{Br} \mathrm{J}$ Ophthalmol. 2010;94(8):1007-11

11. Taylor K, Powell C, Hatt SR, Stewart C. Interventions for unilateral and bilateral refractive amblyopia. Cochrane Database Syst Rev. 2012;4:44.

12. Holmes JM, Lazar EL, Melia BM, Astle WF, Dagi LR, Donahue SP, et al. Effect of age on response to amblyopia treatment in children. Arch Ophthalmol. 2011;129(11):1451-7.

13. Scheiman MM, Hertle RW, Beck RW, Edwards AR, Birch E, Cotter SA, et al. Randomized trial of treatment of amblyopia in children aged 7 to 17 years. Arch Ophthalmol. 2005;123(4):437-47.

14. Pediatric Eye Disease Investigator Group (PEDIG). Patching vs atropine to treat amblyopia in children aged 7 to 12 years a randomized trial. Arch Ophthalmol. 2008;126(12):1634-42.

15. British and Irish Orthoptic Society. Competency Standards and Professional Practice Guidelines
[Internet]. 2014. https://orthoptics.org.uk/ Resources/Documents/Standards/BIOS. Accessed 13 Jan 2018.

16. Repka MX, Wallace DK, Beck RW, Kraker RT, Birch EE, Cotter SA, et al. Two-year follow-up of a 6-month randomized trial of atropine vs patching for treatment of moderate amblyopia in children. Arch Ophthalmol. 2005;123(2):149-57.

17. Ansons AM, Davis H. Diagnosis and management of ocular motility disorders, 4th edn. Hoboken: Wiley-Blackwell. 2014.

18. Moher D, Liberati A, Tetzlaff J, Altman DG, Grp P. Preferred reporting items for systematic reviews and meta-analyses: The PRISMA statement. PLoS Med. 2009;6(7):6.

19. Glanville JM, Lefebvre C, Miles JNV, CamossoStefinovic J. How to identify randomized controlled trials in MEDLINE: ten years on. J Med Libr Assoc. 2006;94(2):130-6.

20. Better Value Healthcare Ltd. Programme CAS. CASP randomised controlled trial checklist [online]. http://docs.wixstatic.com/ugd/dded87 4239299b39f647ca9961f30510f52920.pdf. Accessed 20 Jan 2018.

21. Menon V, Shailesh G, Sharma P, Saxena R. Clinical trial of patching versus atropine penalization for the treatment of anisometropic amblyopia in older children. J AAPOS. 2008;12(5):493-7.

22. Bokinni Y, Shah N, Maguire O, Laidlaw DAH. Performance of a computerised visual acuity measurement device in subjects with age-related macular degeneration: comparison with gold standard ETDRS chart measurements. Eye. 2015;29(8):1085-91.

23. Wilson ME. Adult amblyopia reversed by contralateral cataract formation. J Pediatr Ophthalmol Strabismus. 1992;29(2):100-2. 